\title{
Le Corbusier (1887-1965): un referente del siglo XX todavía presente en las aulas del siglo XXI
}

\author{
Le Corbusier (1887-1965): A model of the $20^{\text {th }}$ century \\ still present in the classrooms of the $21^{\text {st }}$ century
}

\author{
Ione Añón Aracil
}

Universitat de València. ione.anon@uv.es

Recibido: 02.05.2019. Aceptado: 12.09.2019

Resumen: En este trabajo se presenta el fenómeno de divulgación y transmisión de conocimientos artísticos, arquitectónicos y técnicos a nivel internacional a través del conjunto de obras de Le Corbusier (1887-1965) del siglo XX gracias a la labor esencial de los traductores. Su obra escrita en francés no solo se tradujo en su momento al español, alemán, inglés, italiano y portugués de Brasil sino que a día de hoy se sigue utilizando en las aulas de las Escuelas de Arquitectura y urbanismo de todo el mundo. Nuestra pretensión es analizar la terminología relacionada con esos conocimientos técnicos, el impacto de los textos de Le Corbusier en el ámbito teórico de la arquitectura de las sociedades modernas partiendo del francés, como lengua origen, al español como lengua meta.

Palabras clave: traducción especializada; arquitectura; Le Corbusier; terminología; enseñanza.

\begin{abstract}
This paper presents the phenomenon of dissemination and transfer of artistic, architectural and technical knowledge at international level through the collection of works by Le Corbusier (1887-1965) thanks to the essential work of translators. His work originally written in French was translated into Spanish, German, English, Italian and Brazilian Portuguese due to the interest raised. Even now, Le Corbusier's work is used in the classrooms of today's Schools of Architecture and Urban Planning around the world. The aim of this paper is to analyse the terminology used in this technical field and the impact of Le Corbusier's written work on the theoretical field of architecture of modern societies. To do so, we will take French as the source language and Spanish as the target language.
\end{abstract}

Keywords: specialised translation; architecture; Le Corbusier; terminology; transfer of knowledge.

》 Añón Aracil, Ione. 2019. "Le Corbusier (1887-1965): un referente del siglo XX todavía presente en las aulas del siglo XXI". Quaderns de Filologia: Estudis Lingüistics XXIV: 377-393. doi: 10.7203/QF.24.16318 



\section{Introducción}

Le Corbusier es sin duda un referente mundial del siglo XX que todavía está presente en las aulas del siglo XXI. Se trata de un visionario que intentó dar respuesta a los problemas habitacionales de la sociedad del periodo de entreguerras a través de postulados que causarían una revolución del concepto de alojamiento y de las ciudades y que junto a otros arquitectos reconocidos de esa época promovieron la vivienda tal y como la conocemos hoy en día.

Le Corbusier (Charles Édouard Jeanneret-Gris) nació en Suiza en 1887. Allí comenzó su formación en artes y oficios y después se orientó a la arquitectura. A los 29 años se trasladó a París y trabajó en el estudio de Auguste Perret, en esa época adoptó el seudónimo Le Corbusier. Más tarde viajó a Alemania para enriquecer su formación arquitectónica; trabajó con Peter Behrens y fue allí donde conoció a Walter Gropius y Ludwig Mies Van Der Rohe. Viajó también por otros países de Europa como Rumanía, Turquía, Grecia e Italia y de fuera de Europa y más tarde se estableció en Paris donde además de arquitecto y teórico fue también profesor. En 1930 adoptó la nacionalidad francesa. Desde entonces, con una notable reputación, trabajó además de en Europa, principalmente en Francia, más allá de sus fronteras: La India, Massachusetts (EE. UU.), Tokio (Japón), Río de Janeiro (Brasil) y Buenos Aires (Argentina).

Parte de su obra arquitectónica de reconocido prestigio internacional fue inscrita como Patrimonio de la Humanidad en la categoría de bien cultural el 15 de marzo de 2016 como "contribución excepcional al Movimiento Moderno" (UNESCO, 2016).

Además de por su obra proyectada y construida, Le Corbusier es un referente en el ámbito teórico; aspecto en el que versa este trabajo. Le Corbusier fue un adelantado de su tiempo, de ideas muy innovadoras que también sembraron polémica en una sociedad que no estaba preparada para entenderlas.

En 1920 fundó junto con Amédée Ozenfant y Paul Dermée L'Esprit Nouveau, una revista de divulgación artística a través de la cual fue publicando una serie de artículos en los que explicaba sus reflexiones acerca de las necesidades de alojamiento de la sociedad de la época marcada su carácter industrial y por los avances tecnológicos y cómo esta debía cambiar su concepción del alojamiento hasta el momento. Esta revista tuvo tal repercusión internacional que Le Corbusier recopiló varios de 
los artículos junto con otras reflexiones y propuestas innovadoras en una de sus obras más importantes Vers une architecture (1923).

En este libro introdujo, entre otros, el concepto de La machine à habiter (La máquina para habitar). Esto es, entender la vivienda como una herramienta que debe ser a la vez funcional y eficaz para vivir. Siendo este un concepto revelador marcado por el racionalismo de la época, que a día de hoy todo el mundo acuña a Le Corbusier.

La importancia internacional de sus contribuciones supuso la necesidad de traducción de su obra a diversos idiomas. Vers une architecture (1923) se tradujo al español, alemán, inglés, italiano y portugués de Brasil. Además de Vers une architecture (1923), de sus más de 50 obras escritas destacan sus contribuciones arquitectónicas de Le modulor (1950) Le modulor II (1955), L'unité d'habitation de Marseille (1950), La Chartre d'Athènes (1943) y Les cinq points de l'architecture (1926). En este trabajo estudiaremos el impacto de estas obras en relación con la terminología empleada y la importancia de la labor del traductor.

No obstante, para entender el porqué de la traducción de la obra de Le Corbusier y la repercusión que tuvo en todo el mundo es necesario contextualizar el momento: la arquitectura moderna. Asimismo, conviene destacar las características del entorno de la traducción y los traductores de la época y por último, analizar la terminología innovadora empleada y el asentamiento o transformaciones que ha sufrido. En los siguientes apartados analizaremos la importancia de cada uno de estos aspectos para que la obra de Le Corbusier, fallecido hace 54 años, siga presente en las aulas del siglo XXI.

\section{La arquitectura moderna}

A finales del siglo XIX surge en Inglaterra el movimiento de Artes y oficios (Arts and Crafts) como respuesta a los productos procedentes de la industria producidos en serie. A principios del siglo XX surge en otros países de Europa el Modernismo (también llamado Art Nouveau en Francia y Bélgica, Jugendstil en Alemania, Sezession en Austria, Estilo Liberty o Floreale en Italia y Modernismo en España).

Se trata de un nuevo estilo o corriente artística que buscaba romper con las formas del pasado, tomando la naturaleza como inspiración y utilizando las técnicas vanguardistas de la revolución industrial como es incorporar el acero y el vidrio en las creaciones. 
De manera paralela en Estados Unidos, la Escuela de Chicago fue pionera en la utilización de los nuevos materiales procedentes de la industria y técnicas de construcción para levantar grandes edificios. Louis Sullivan, uno de sus máximos representantes acuñó el lema form follows function (la forma sigue a la función) que más tarde se convirtió en el lema de la arquitectura moderna.

Como respuesta al gran incendio de Chicago de 1871 que destruyó gran parte de la ciudad, la Escuela de Chicago apostó por una propuesta urbanística de edificación en altura. Para ello utilizaron estructuras metálicas sobre cimientos de hormigón armado, ventanas corridas y fábrica de ladrillo para las fachadas y ascensores eléctricos para el desplazamiento vertical de los usuarios. Esto resultó en la construcción de los primeros rascacielos.

Con respecto al alojamiento de las clases medias, se desarrolló también una arquitectura residencial orgánica donde primaba la utilización de madera y piedra. El principal exponente de la arquitectura orgánica fue Frank Lloyd Wright, quien trabajó en el estudio Louis Sullivan y fue uno de los principales maestros de la arquitectura del siglo XX.

En Alemania, se fundó en 1907 la Deutscher Werkbund. Se trataba de una asociación de arquitectos, artistas en industriales, entre los que destacan Hermann Muthesius, Peter Behrens y Henry van de Velde, que pretendía combinar las artes y oficios tradicionales con las técnicas industriales de producción en masa para que Alemania fuera competitiva frente a potencias como Gran Bretaña y Estados Unidos y obtener así productos producidos en serie con unos estándares adecuados de calidad y diseño. Asimismo, la Deutscher Werkbund fue precursora del diseño industrial, del diseño gráfico y de la creación de la Bauhaus.

En 1919, Walter Gropius miembro de la Deutscher Werkbund_decidió fundar una escuela de arte, diseño y arquitectura a la que llamó Staatliches Bauhaus y a la que se conoce como Bauhaus tras el final de la Primera Guerra Mundial. Bauhaus proviene de la unión de las palabras Bau que significa 'construcción' en alemán y Haus que significa 'casa'. En esta escuela se enseñaba a los estudiantes a utilizar los nuevos materiales y técnicas para crear edificios, muebles y objetos funcionales y originales. Algunos de sus profesores más célebres además de los directores Walter Gropius y Ludwig Mies Van der Rohe fueron: Paul Klee, Wassily Kandinsky, Josef Albers, Johannes Itten, Hannes Meyer, Marcel Lajos Breuer y László Moholy-Nagy. 
Su sede originalmente en Weimar cambió de Weimar a Dessau por la ocupación nazi y por último, a Berlín, que finalmente también fue cerrada en 1933 por los nazis. Tras el cierre, muchos de sus integrantes emigraron a Estados Unidos donde continuaron con la metodología Bauhaus hasta el periodo de la Guerra Fría. Uno de los principios establecidos en la Bauhaus desde su fundación fue la forma sigue a la función. Este principio marcó un antes y un después en la concepción de la arquitectura y el diseño.

La influencia de la Bauhaus junto con el conjunto de corrientes y estilos artísticos de principios del siglo XX en todo el mundo formaron las bases de lo que se conoce como arquitectura moderna. La arquitectura moderna surge como respuesta a los problemas que la había generado la revolución industrial. Los precursores estudian y plantean fórmulas para organizar las ciudades desde el punto de vista del urbanismo y se centran en las necesidades del hombre en relación al trabajo, vivienda, transporte y ocio. La arquitectura moderna se caracteriza por una ruptura con composición clásica, la simplificación de las formas, el rechazo al ornamento, la búsqueda del minimalismo, la utilización de las nuevas tecnologías de la construcción con el uso del acero, vidrio y hormigón armado y la idea de que la forma sigue a la función.

En 1928 se fundó el CIAM (Congrès International d'Architecture Moderne) (Congreso Internacional de Arquitectura Moderna) en el castillo de la Sarraz en Suiza, por un grupo de 28 arquitectos organizado por Le Corbusier, Hélène de Mandrot (propietaria del castillo) y Sigfried Giedion (primer secretario general). El CIAM tenía 6 comisiones permanentes, una de las cuales presidía Le Corbusier y tenía como vicepresidente a José Luis Sert, y organizaba reuniones y congresos en toda Europa con el objetivo de compartir y debatir los principios del movimiento moderno desde el punto de vista del funcionalismo.

El CIAM fue muy influyente en todo el mundo. De hecho, tras la Segunda Guerra Mundial se utilizaron los criterios de La Chartre d'Athènes (Le Corbusier, 1943) (Carta de Atenas), un manifiesto urbanístico que recoge las conclusiones del cuarto congreso del CIAM realizado en 1933 para reconstruir algunas de las ciudades. La Carta de Atenas sentaba las bases para la zonificación de las ciudades siguiendo criterios funcionales en cuanto a la vivienda, el trabajo, el transporte y el ocio y para los edificios residenciales disponiendo que estos debían situarse en los mejores entornos, no se debían construir a lo largo de 
vías de comunicación, se debía promover la edificación en altura con la utilización de las nuevas técnicas constructivas para crear 'tapices verdes', debían respetarse los aspectos relacionados con la higiene y el control del asoleamiento.

La arquitectura moderna o movimiento moderno también fueron acuñados con el término Estilo Internacional a partir de la exposición organizada en el Museo de Arte Moderno de Nueva York en 1932 por Henry-Russell Hitchcock y Philip Johnson y en el libro publicado por ambos The International Style: Architecture since 1922.

Aunque la arquitectura moderna no tiene un comienzo determinado, globalmente se reconoce a Le Corbusier, Walter Gropius y Ludwig Mies van der Rohe como los precursores del movimiento moderno. Por otro lado, destaca la figura estadounidense Frank Lloyd Wright de la corriente organicista, pese a que no se veía a sí mismo encasillado dentro de la arquitectura moderna de los arquitectos modernos europeos.

\section{Interés creciente. Necesidad de traducción}

Como se ha visto en el apartado anterior, desde principios del siglo XX hasta los años sesenta fueron unos años muy activos en diversas partes del mundo desde el punto de vista artístico. Esto se debe a la evolución de la técnica gracias a la industria y al interés creciente de los arquitectos, artistas y diseñadores en adaptar los productos, las viviendas y las ciudades a las necesidades de la sociedad y romper con lo anterior. Por otro lado, los nuevos sistemas constructivos tenían un componente económico y de rapidez en las fases de ejecución que interesaba también a los políticos y empresarios.

Desde el punto de vista histórico, en el periodo de entreguerras el movimiento moderno comenzó a despertar gran interés que culminó tras la Segunda Guerra Mundial. De ahí, los numerosos viajes que realizó Le Corbusier, al igual que otros arquitectos y artistas, por Europa, Asia, Estados Unidos y Latino América.

Con respecto al ámbito de la traducción, esta expansión del movimiento moderno junto con los viajes, congresos y los CIAM que realizaron las figuras de relevancia resultan de gran interés porque crearon la necesidad de traducir, ya que era la única manera de estar informado de los últimos avances. Las obras teóricas como la de Le Corbusier cobraron cierta importancia, pues proponían una forma nueva de habitar y 
de vivir a partir de una nueva manera de concebir las necesidades de la sociedad y de cómo la ciudad debía comportarse para satisfacer dichas necesidades.

Argentina, fue uno de esos lugares que se contagió del racionalismo del movimiento moderno y en concreto de la obra de Le Corbusier. En 1929, Le Corbusier visitó Argentina y propuso una serie de ideas urbanísticas que más tarde materializó en el Plan Directeur, Buenos Aires, Argentine (1936).

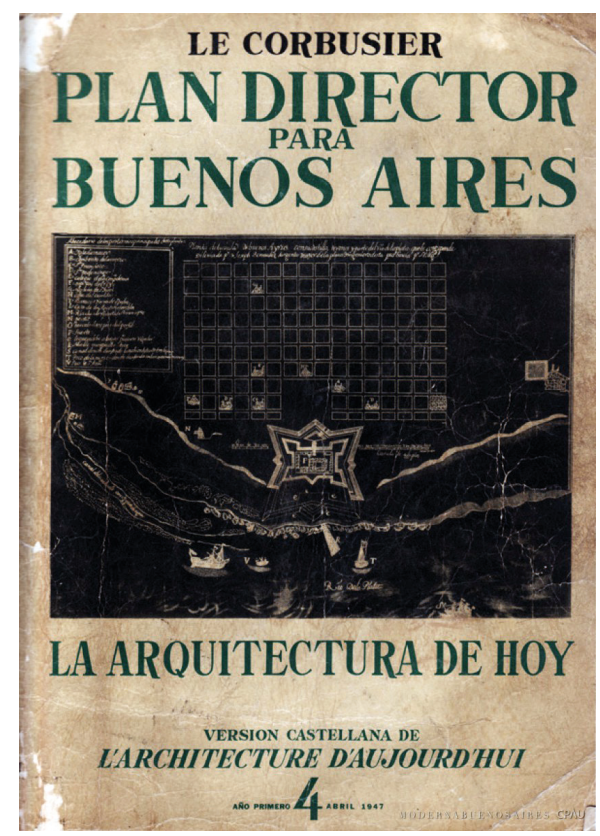

Figura 1. Portada versión castellana de Plan director para Buenos Aires, Le Corbusier

El interés por la arquitectura moderna y el movimiento moderno fomentó la creación de escuelas y facultades de arquitectura, urbanismo y diseño en Argentina. Entre 1920 y 1970 se crearon once facultades y escuelas de arquitectura, urbanismo y diseño, seis de ellas en Universidades Nacionales de las grandes ciudades y las restantes en universidades privadas. Tanto es así que el complejo universitario de la Universidad de Buenos Aires que se construyó en 1960 se inspiró en las propuestas de Le Corbusier de 1929. 
Con esta perspectiva no es de extrañar que las primeras traducciones al español se realizaran en Buenos Aires. Por ejemplo, Vers une architecture, (1923) se tradujo y publicó en español por primera vez en 1939 por Luis A. Romero, en Buenos Aires a través de la editorial El Distribuidor Americano; Le modulor, (1948) se tradujo y publicó en español por Rosario Vera, en Buenos Aires en 1953 a través de la editorial Poseidón y Le modulor II (1953) se tradujo y publicó en español por Albert Junyent, en Buenos Aires en 1962 a través de la editorial Poseidón.

En España también llegó la influencia del movimiento moderno y propició un interés por la arquitectura que favoreció la creación de nuevas escuelas de arquitectura universitarias. Hasta los años cincuenta solamente existían dos escuelas de arquitectura fundadas en el siglo XIX en Madrid y Barcelona. Sin embargo, desde los años cincuenta hasta finales de los años ochenta se abrieron ocho escuelas más repartidas por toda España: Sevilla, Valencia, Valladolid, La Coruña, Las Palmas de Gran Canaria, Vallés, San Sebastián y Alicante. Fueron de creación un poco más tardía que en Argentina y otros lugares de mundo posiblemente debido a la situación política de España de ese periodo.

En este contexto, aparece también la necesidad de aprender de los maestros del movimiento moderno, y por tanto, las traducciones publicadas en España. Por ejemplo, Vers une architecture, (1923) se tradujo y publicó en español por Josefina Martínez Alinari en 1977, en Barcelona, a través de la editorial Poseidón (versión con la que se trabaja en este trabajo). De igual forma sucedió con la segunda y tercera edición de 1978. Con respecto a Le modulor, (1948), la versión castellana de Rosario Vera se reeditó una segunda y tercera vez, en 1979 y 1980 respectivamente, en Barcelona a través de la editorial Poseidón.

El interés por la obra teórica de Le Corbusier ha estado presente tanto en el contexto formativo de la enseñanza universitaria como en el ámbito profesional hasta la actualidad. Prueba de ello es que una de las últimas ediciones de las obras mencionadas data de 2016. El estudio de Le Corbusier forma parte del plan de estudios de la titulación de arquitectura en los contenidos de la rama de historia de la arquitectura, por lo que todos los profesionales han estudiado a Le Corbusier como estudiantes y han aprendido de él.

Aunque en la actualidad la traducción especializada está extendida y diversificada, los traductores de la época tuvieron que hacer frente a 
la demanda creciente sin ser especialistas en la materia. Por esta razón, el resultado de las primeras traducciones, como analizaremos en el siguiente capítulo es una traducción más bien literal de los textos origen.

\section{Terminología}

Como ya se ha mencionado, la contribución teórica de le Corbusier destaca por sus novedosas aportaciones conceptuales. Aunque el lenguaje general de su obra escrita no presenta dificultades especiales son los nuevos conceptos los que suponen un reto para los traductores del momento. Traductores que no dominan necesariamente la materia porque no existía el recorrido en lenguaje de especialidad, esto es, la traducción especializada.

Por esta razón, ante unos planteamientos tan novedosos y sin poder tener referencias similares en relación con la terminología, los traductores apostaron por utilizar la traducción literal en la mayoría de los casos. A continuación, destacamos algunos de los conceptos a los que nos referimos utilizando la versión original de Vers une architecture, (1923) y la traducción al castellano de Josefina Martínez Alinari de 1977.

\subsection{La machine à habiter}

(...) il faut donc transformer totalement sur les usages en honneur chez MM., les architectes, tamiser le passé et tous ses souvenirs à travers les mailles de la raison, poser le problème comme se le sont posé les ingénieurs de l'aviation et construire en série des machines à habiter (1923:100).

(...) hay que transformar totalmente los métodos de los arquitectos, tamizar el pasado y todos los recuerdos a través de las mallas de la razón, plantear el problema como se lo han planteado los ingenieros aeronáuticos y construir en serie las máquinas de habitar (1977:100).

Le Corbusier estaba fascinado con el desarrollo de las máquinas en el ámbito de la ingeniería, en concreto los aviones y los automóviles. Por esta razón, concebía las viviendas como máquinas para habitar, término que la traductora tradujo literalmente y que ha sido mayoritariamente aceptado por los especialistas; los arquitectos. Es un términoconcepto que se asocia con Le Corbusier y el movimiento moderno, 
además contiene 23.100 resultados en internet. Asimismo, aparece la idea de la producción en serie que veremos más adelante.

\subsection{Les villes-tours}

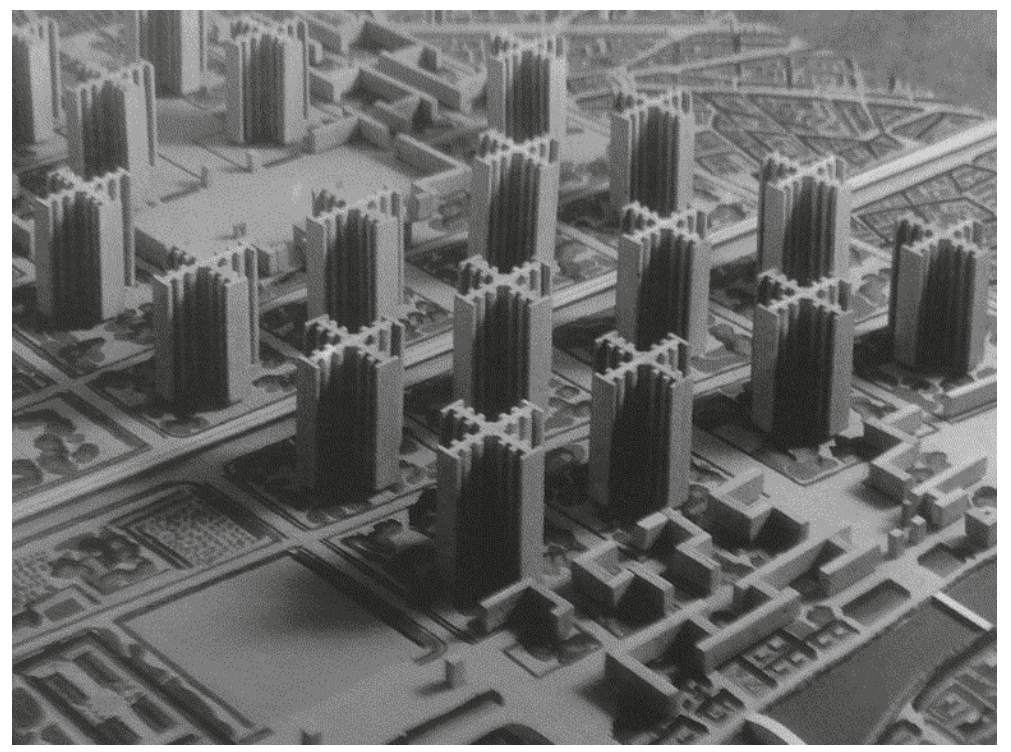

Figura 2. Les Villes-Tours

LES VILLES-TOURS. Proposition de Lotissement. Soixante étages, hauteur 220 mètres; distance entre tours 250 à $300 \mathrm{~m}$ (équivaut à la largeur du Jardin des Tuileries). (...) Il semble que de telles constructions doivent être consacrées exclusivement aux affaires (bureaux) et par conséquent élevées au centre des grandes villes dont elles décongestionneraient les artères : la vie de famille s'adapterait mal au mécanisme prodigieux des ascenseurs. Les chiffres sont effarants et sans pitié, magnifiques: en accordant à chaque employé une superficie de $10 \mathrm{~m}^{2}$, un gratte-ciel de $200 \mathrm{~m}$ de large abriterait 40.000 personnes (...) (1923: 42).

LAS CIUDADES-TORRES. Proposición de loteo. Sesenta pisos, de una altura de 250 a 300 metros (equivalente a la extensión del Jardín de las Tullerías). (...) Al parecer tales construcciones deben estar consagradas 
exclusivamente al comercio (oficinas) y por consiguiente levantadas en el centro de las grandes ciudades, cuyas arterias descongestionarían. La vida familiar se adaptaría mal al mecanismo prodigioso de los ascensores. Las cifras son espantosas e implacables, magníficas; concediendo a cada empleado una superficie de $10 \mathrm{~m}^{2}$, un rascacielo de 200 metros albergaría a 40.000 personas (...) (1977: 42).

Le Corbusier influenciado por el término Villes-Tours de Auguste Perret idea su propio concepto de Villes-Tours como ciudad ideal con rascacielos de planta cruciforme y amplias zonas verdes en planta baja que más tarde propondría en el Plan Voisin de 1922 para la ciudad de Paris y recogería en su obra La ville radieuse de 1934. Para este nuevo concepto con cierta influencia de los rascacielos americanos la traductora apuesta por una traducción literal, ya que no se identifica con ningún otro tipo de edificación existente. Aunque en la actualidad sí se identifica el término ciudades-torres con Le Corbusier no es un concepto que se utilice. Sin embargo, cabe destacar que su contribución propició un nuevo concepto de ideal de ciudad: la ciudad vertical que desarrolló Hilberseimer, un coetáneo de la época, como mejora de la ciudad ideal de Le Corbusier. Asimismo, cabe destacar que el concepto de ciudad vertical se ha reinventado hasta la actualidad y se sigue estudiando como modelo de sostenibilidad en las ciudades.

Por otro lado, en la cita se encuentra proposition de lotissement que la traductora tradujo por proposición de loteo. Aunque la Real Academia Española recoge el término loteo como Acción y efecto de lotear, entendiendo lotear como dividir en lotes, generalmente un terreno, el uso más extendido del término lotissement en español es urbanización o parcelación. De igual manera sucede con proposition, cuyo uso más extendido en español, al menos desde el punto de vista del urbanismo, es el de propuesta en lugar de proposición. Asimismo cabe destacar la traducción de gratte-ciel por rascacielo (incorrecto en español) en lugar de rascacielos. Posiblemente se trate de un error de tipo tipográfico, ya que más hacia delante en el texto aparece el rascacielos norteamericano. No obstante, es importante considerar estas pequeñas apreciaciones porque no debemos olvidar que en ese momento la tipología de rascacielos era relativamente nueva y el concepto estaba asentándose en nuestra cultura. 


\subsection{La conception du logis}

Si, pourtant, la paix pose maintenant le problème : le Nord à reconstruire. Mais, voilà: on est totalement désarmé, on ne sait pas bâtir moderne, matériaux, systèmes constructifs, conception du logis. Les ingénieurs étaient occupés aux barrages, (...) Les architectes dormaient (1923: 88).

Sin embargo, ahora es la paz la que plantea el problema: hay que reconstruir el norte. Pero nos hallamos totalmente desarmados, ya no se sabe construir con lo moderno: materiales, sistemas de construcción,

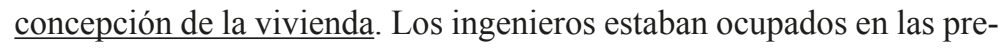
sas de contención, (...) Los arquitectos dormían (1977: 88).

En este extracto se puede apreciar la importancia de la obra teórica de Le Corbusier en cuanto a movilización de los arquitectos y crítica con lo realizado hasta el momento. Le Corbusier apuesta por una nueva concepción de la vivienda como propone Josefina Martínez con su traducción. Aunque no se trata de un término complicado sí resulta una manera de pensar novedosa y también forma parte del lenguaje de especialidad del campo de la arquitectura.

A raíz de esta idea de vivienda, Le Corbusier anima a las masas a exigir a sus caseros lo que él considera unos mínimos en las viviendas y propone un Manuel de l'habitation (Manual de la vivienda).

Pourquoi n'exigez-vous pas de votre propriétaire. $1^{\circ}$ Les casiers à linge de corps et vêtements dans votre chambre à coucher, le tout à une seule profondeur, à hauteur humaine, et pratiques comme une malle « Innovation $» 2^{\circ}$ Dans votre salle à manger, les casiers à vaisselle,(...) fermant bien et avec assez de tiroirs pour que vote « rangement $»$ soit en un tourne-main (...) (1923: 91).

Por qué no exigís de vuestro casero: $1^{\circ}$ Placares para ropa blanca y trajes, en vuestros dormitorios, todos de la misma profundidad, de altura humana y prácticos como un baúl "Innovation” $2^{\circ}$ En vuestro comedor, armarios para la vajilla, (...) que cierren bien y con los cajones suficientes para que la "colocación" se haga en un abrir y cerrar de ojos (...) (1977: 91). 
Josefina Martínez transcribe sin traducir Innovation tal y como aparece en el texto origen; sin embargo, sí traduce rangement por colocación. No obstante, la metodología traductológica no siempre es igual a lo largo de la obra. Es el caso de, “(...) des casiers pour ranger vite chaque chose à sa « right place »". En el texto origen se encuentra escrito en inglés; sin embargo, en la versión castellana aparece traducido, "(...) lugares donde poner rápidamente cada cosa en el sitio adecuado".

Por otro lado, cabe destacar que en el extracto anterior se realiza un guiño a través de à hauteur humaine al tema de la relación de las proporciones humanas que Le Corbusier estudió en profundidad en sus obras de Le Modulor (1950). También aparece otra referencia a l'échelle humaine en capítulos anteriores de Vers une architecture (1923).

\subsection{Maisons « Domino »}

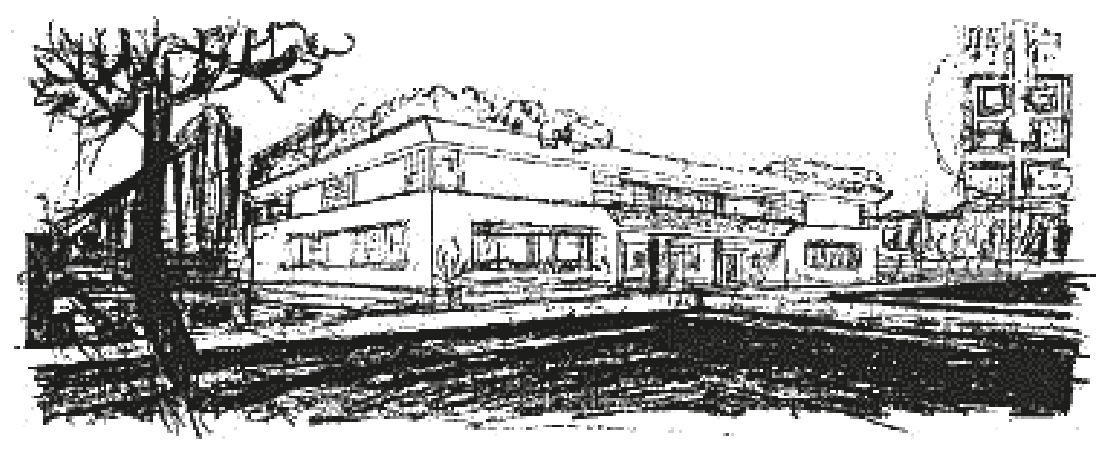

Figura 3. Maison Domino

1915. Maison « Domino ». Le procédé constructif est appliqué ici à une maison de maître qui est établie au prix de cube de la simple maison ouvrière. Les ressources architecturales du procédé constructif autorisent des dispositions larges et rythmées et permettent de faire de véritable architecture. C'est ici que le principe de la maison en série montre sa valeur morale : un certain lien commun entre l'habitation du riche et celle du pauvre, une décence dans l'habitation du riche (1923: 194).

1915. Casa 'Domino'. Aquí el procedimiento de construcción es aplicado a una casa señorial cuyo precio es el cubo del de la simple casa obrera. Los recursos arquitectónicos del procedimiento constructivo 
autorizan disposiciones amplias y rítmicas y permiten hacer arquitectura verdadera. Aquí el principio de la casa en serie muestra su valor moral: un cierto lazo común entre la vivienda del rico y la del pobre, una decencia en la morada del rico (1977: 194)

La Casa 'Domino' es de por sí un concepto nuevo sistema de construcción de viviendas en serie que introdujo Le Corbusier. Josefina Martínez optó por una traducción literal al no existir otra referencia similar. Aunque el término es ampliamente acuñado a Le Corbusier, cabe mencionar que existe también otra posible nomenclatura como es 'casa DOM-INO'.

La evolución del sistema Domino supuso que Le Corbusier junto con Pierre Jeanneret escribieran en 1926 un manifiesto arquitectónico que recogía los cinco principios que dictaban su técnica: Les cinq points d'une nouvelle architecture; la cual se materializó en la obra construida la Villa Savoye. Estos cinco principios son: les pilotis (pilotis), le toitterrasse (techo-jardín o terraza jardín), le plan libre (planta libre), la fenêtre-bandeau (ventana horizontal o ventana alargada) y la façade libre (diseño libre de fachada o fachada libre).

Aunque existen varias propuestas de traducción, todas ellas giran en torno al mismo concepto. Es importante recalcar que el concepto de pilotis no solo ha sido ampliamente extendido y aceptado en el campo de la arquitectura de lengua española sino que el término se ha mantenido también lengua inglesa, alemana e italiana, con independencia de que todas las lenguas cuentan con un término equivalente.

Por otro lado, con respecto a la obra Le Modulor, tanto en Le Modulor I (1950) como Le Modulor II (1955), es importante recalcar que aunque la terminología no resultaba especialmente compleja, la traducción de la obra completa sí fue de especial importancia al tratarse de un Ensayo sobre una medida armónica a la escala humana aplicable universalmente a la arquitectura y a la mecánica que establecía un sistema de medidas basado en las proporciones humanas en que cada magnitud está relacionada con la anterior con el número Áureo. Esta proporción servía para el diseño de viviendas, mobiliario, etc.

En 1946, en un viaje que realizó Le Corbusier a Nueva York con motivo de la construcción de la sede de Naciones Unidas en Estados Unidos tuvo "el placer de conversar largamente sobre el Modulor con el profesor Albert Einstein en Princetown" (E1 Modulor, versión 
castellana). Le Corbusier explica en su obra escrita que lo explicó mal y con falta de claridad, pero que Einstein empezó a calcular y él lo interrumpió 'estúpidamente'. Sin embargo, esa misma tarde Einstein le escribió: "c'est une échelle de proportion qui rend le mauvais difficile et le bon facile” (Le Modulor, 1950: 55) que Rosario Vera tradujo por "es una gama de proporciones que hace lo malo difícil y lo bueno fácil".

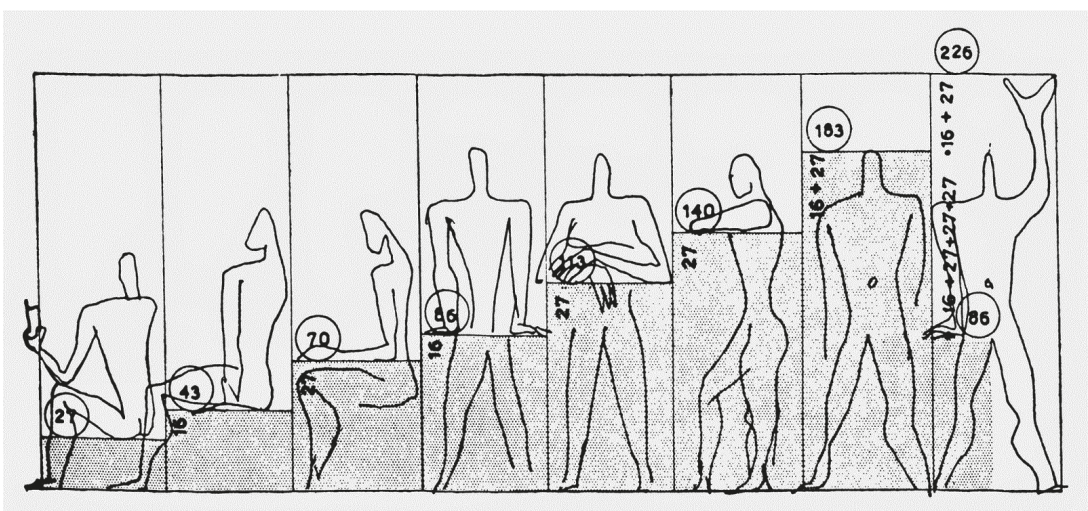

Figura 4. Estudio de El Modulor

\section{Conclusiones}

Como hemos analizado en este trabajo, la obra teórica escrita de Le Corbusier fue y sigue siendo un referente de la arquitectura moderna en todo el mundo. Por este motivo, a mediados del siglo XX se realizaron las primeras traducciones a diversos idiomas. Estas traducciones no las realizaron traductores expertos en arquitectura porque no existía la traducción especializada todavía; sin embargo, a partir del contexto, de los dibujos e ilustraciones de Le Corbusier, así como con la utilización de la técnica de traducción literal supieron trasladar los conceptos novedosos que incluía Le Corbusier en sus obras a todos los rincones del mundo. De hecho, algunos de estos conceptos como pilotis y màchine à habiter perduran en el tiempo y otros como villes-tours han evolucionado.

El estudio de su obra comenzó ya en la enseñanza universitaria de las escuelas de arquitectura de principios y mediados del siglo XX. Desde los años veinte hasta los años ochenta la creación de escuelas de 
arquitectura creció exponencialmente, debido en cierto modo a la estabilidad política, la concienciación social e intelectual y el desarrollo de la industria. En el siglo XXI los estudiantes de arquitectura de todo el mundo siguen estudiando la obra teórica de Le Corbusier, ya que forma parte del Plan de estudios, lo que supone que se sigan reeditando gran parte de sus obras y algunas de ellas sean susceptibles de nuevas traducciones.

\section{Bibliografía}

Cabré i Castellví, M. Teresa. 1999. La terminología: representación y comunicación. Elementos para una teoría de base comunicativa y otros artículos (ej. 2. ${ }^{\mathrm{a}}$ ed.). Documenta Universitaria.

Le Corbusier. 1924 Vers une architecture, Nouvelle édition revue et augmentée.

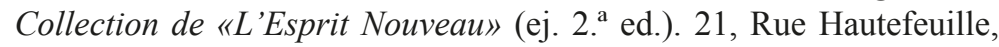
21, Paris: Les éditions G. Crès et C.

Le Corbusier. Traducción: Martínez Alinari, Josefina. 1977. Hacia una arquitectura ([1. ${ }^{a}$ ed. 1977, 2. ${ }^{a}$ ed. 1978] 1998). Barcelona: Ediciones Apóstrofe, Colección Poseidón.

Le Corbusier. Traducción: Vera, Rosario. 1953. El Modulor (ej. 2.. ed.). Buenos Aires: Editorial Poseidón.

Paulin, Michel. 2001. Guide Technique: Vocabulaire illustré de la construction. Illustrated vocabulary of construction. Français-Anglais (ej. 2. ed.). España: Editions Le Moniteur. 